\title{
Evaluación económica y financiera de las Cooperativas de Ahorro y Crédito del Ecuador. Caso Segmento 2 del cantón Ambato provincia de Tungurahua.
}

Economic and financial evaluation of the Savings and Credit Cooperatives of Ecuador. Case Segment 2 of the canton Ambato province of Tungurahua.

José Fabián Fonseca Vasconez. ${ }^{1}$, Angelita Genoveva Tapia Bonifaz. ${ }^{2}$, Sandra Patricia Jácome Tamayo. ${ }^{3}$ \& Víctor Gabriel Avalos Peñafiel. ${ }^{4}$

\begin{abstract}
.
DOI: https://doi.org/10.33262/cienciadigital.v3i3.2.718

The Ecuadorian Financial System is the set of financial institutions regulated by the Superintendency of Banks and the Superintendency of Popular and Solidarity Economy; which enable the various movements of money made by natural and legal persons, be they savings, investment, and credit; in this way the National Financial System contributes to the development of the country, strengthening productive investment and responsible consumption; however, the historical background of the Ecuadorian financial system shows the instability of the sector, especially the cooperative, which has not been consolidated until now, despite the existence of a dollarized economy and the establishment of specialized control entities in the area; problematic that has given rise to the generation of socioeconomic disadvantages; In this sense, the present investigation has as objective to evaluate the economic and financial situation of the Cooperatives of Savings and Credit of Ecuador. Case Segment 2 of the canton Ambato province of Tungurahua, for which methods were used: theoretical (analysis-synthesis, inductive-deductive and historical-logical); empirical:
\end{abstract}

1 Universidad Técnica Estatal de Quevedo, Facultad de Ciencias Empresariales, Quevedo, Ecuador, jfonsecav@uteq.edu.ec

${ }^{2}$ Escuela Superior Politécnica de Chimborazo, Facultad de Administración de Empresas, Riobamba, Ecuador, genoveva.tapia@espoch.edu.ec

${ }^{3}$ Escuela Superior Politécnica de Chimborazo, Facultad de Administración de Empresas, Riobamba, Ecuador, sandra.jacome@espoch.edu.ec

${ }^{4}$ Escuela Superior Politécnica de Chimborazo, Facultad de Administración de Empresas, Riobamba, Ecuador, victor.avalos@espoch.edu.ec 
(qualitative and quantitative), which allowed to understand facts and relevant phenomena throughout the research process. Finally, the conclusions of the study are exposed, which may promote new questions susceptible to future research.

Keywords: Financial system, savings and credit cooperatives, profitability, solvency, liquidity.

\section{Resumen.}

El Sistema Financiero ecuatoriano, es el conjunto de instituciones financieras reguladas por la Superintendencia de Bancos y por la Superintendencia de Economía Popular y Solidaria.; las cuales viabilizan los diversos movimientos de dinero que realizan personas naturales y jurídicas, sean estos ahorro, inversión, y crédito; de esta manera el Sistema Financiero Nacional contribuye al desarrollo del país, fortaleciendo la inversión productiva y el consumo responsable; sin embargo, los antecedentes históricos del sistema financiero ecuatoriano evidencia la inestabilidad del sector, especialmente el cooperativo, el mismo que no ha logrado consolidarse hasta la actualidad, a pesar de existir una economía dolarizada y la instauración de entidades de control especializadas en el área; problemática que ha dado lugar a la generación de inconvenientes socioeconómicos; en este sentido, la presente investigación tiene como objetivo evaluar la situación económica y financiera de las Cooperativas de Ahorro y Crédito del Ecuador. Caso Segmento 2 del cantón Ambato provincia de Tungurahua, para lo cual se utilizó métodos: teóricos (análisis-síntesis, inductivo-deductivo e histórico-lógico); empíricos: (cualitativos y cuantitativos), los cuales permitieron comprender hechos y fenómenos relevantes durante todo el proceso de investigación. Finalmente, se exponen las conclusiones del estudio las mismas que pueden promover nuevas interrogantes susceptibles de investigaciones futuras.

Palabras claves: Sistema financiero, cooperativas de ahorro y crédito, rentabilidad, solvencia, liquidez.

\section{Introducción}

El sistema financiero del Ecuador se ha caracterizado por tener problemáticas entorno a su rentabilidad, solvencia, y liquidez, e incluso jurídicas, el mismo que entra en recesión severa a partir del 8 de marzo de 1999 al cerrarse las operaciones financieras por cinco días, suceso al que se denominó feriado bancario, cuyo desenlace fue la quiebra y liquidación de algunas instituciones financieras.

Problemáticas socioeconómicas neurálgicas que impidió que los clientes recuperarán sus depósitos como consecuencia del pánico generalizado en todo el sistema financiero nacional; 
evento que estuvo acompañado de una inflación del 56\% el cual llego al 97\%, ya en pleno proceso de dolarización, la cual cayó sistemáticamente desde el año 2001 hasta colocarse en el 1,95\% el año 2003 la primera de un dígito desde 1980 tal como lo detalla diario El Comercio en su edición del 29 de mayo de 2014.

A partir de la dolarización la economía ecuatoriana ha tendido a crecer de forma sostenida como producto del incremento de las remesas, y la tendencia creciente del precio del barril de petróleo, lo cual ha impulsado el incremento del PIB a nivel nacional, cuyo efecto multiplicador ha generado una tendencia creciente promedio del $2.96 \%$ en el sector cooperativo financiero, con ello se promovió un alto nivel de competitividad entre las mismas; sin embargo también han surgido problemas neurálgicos para este sector financiero, siendo afectada, su liquidez, solvencia, rentabilidad e imagen corporativa.

En este sentido, a partir del año 2008 con la creación y aprobación de la nueva Constitución del Ecuador se determina la Economía Popular y Solidaria como modelo económico que regirá en el país, el cual dio lugar a la generación de nuevas normativas e instituciones para el control especializado del sistema financiero ecuatoriano; estableciendo que la Superintendencia de Bancos (SB) será la encargada de control de los Bancos y la Superintendencia de Economía Popular y Solidaria (SEPS) de las Cooperativas de Ahorro y Crédito, Cajas de Ahorro, Bancos Comunales y Cajas Comunales; determinándose en aquel entonces que los bancos tienen mayor participación del sistema financiero nacional con el $84 \%$, y las Cooperativas de Ahorro y Crédito (COAC) cuentan con un $11 \%$ de participación, y las otras instituciones financieras con el $5 \%$ promedio.

En este ámbito, la SEPS promueve el Catálogo Único de Cuentas del sistema financiero cooperativo ecuatoriano, con el objetivo de generar un control contable eficiente el cual conlleve a la rendición de cuentas de los directivos hacia los organismos de control, so cios y miembros de las organizaciones financieras, lo cual ha generado procesos ineficientes antes que eficientes. Problemática neurálgica que ha sido causal de la liquidación de algunas instituciones del sector financiero cooperativo en consideración que según el boletín financiero emitido el 31 de diciembre del 2014 por la SEPS con el informe del 2018 a la misma fecha, se determina que se han liquidado en 4 años más de 340 COAC.

Actualmente, según la SEPS (2019), las COAC constituyen el grupo de mayor tamaño en términos de activos, pasivos y patrimonio, respecto al total nacional de cooperativas existentes en el Ecuador; los activos de las COAC ascendieron a 5295,8 millones de dólares, es decir el $93 \%$ del total del sector cooperativo; en los últimos 5 años los activos de las cooperativas de ahorro y crédito crecieron en 73\%, razón por la cual este sector financiero ha logrado una participación en el mercado del 11,29\% en relación al Producto Interno Bruto (PIB). 
En este mismo ámbito, por cada USD 100 que la banca privada otorga en créditos al sector rural, las COAC colocan USD 250, de lo cual el 76\% de colocación de créditos se otorga a socios que tienen instrucción primaria y secundaria, generalmente para impulsar emprendimientos; destacándose además que el 30.6\% de los ecuatorianos son socios de alguna COAC, por tanto, a nivel nacional el número de socios bordea los 4,6 millones de personas, datos proyectado a partir del Censo Económico (INEC, 2010), proyectado a la actualidad.

En esta misma línea, las COAC están clasificadas por segmentos según la valoración de los activos tal como se muestra la Tabla 1.

Tabla1: segmentación de las COAC

\begin{tabular}{ll}
\hline Segmentos & \multicolumn{1}{c}{ Activos (USD) } \\
\hline 1 & Mayor a 80'000.000,00 \\
2 & Mayor a 20'000.000,00 hasta 80'000.000,00 \\
3 & Mayor a 5'000.000,00 hasta 20'000.000,00 \\
4 & Mayor a 1'000.000,00 hasta 5'000 000,00 \\
5 & Hasta 1'000.000,00 \\
& Cajas de Ahorro, bancos comunales y cajas comunales \\
\hline
\end{tabular}

Fuente: Informe para la clasificación SEPS (2019)

El primer inventario realizado por la SEPS (2012) determino que existía 946 COAC, actualmente existe 598 es decir han quebrado 348 COAC $36.78 \%$ menos de las cuales 27 pertenecen al primer segmento, 38 al segundo segmento, 86 al tercer segmento, 180 al cuarto segmento y 267 al quinto segmento; en este sentido el segmento tres, cuatro y cinco agrupan al $96 \%$ del total de COAC a nivel nacional, sin embargo el segmento uno y dos concentran el $88,66 \%$ del total de activos y el $84,51 \%$ del total de socios del sector de COAC.

La mayor cantidad de COAC se encuentra localizadas en la provincia del Pichincha $20 \%$, seguidas por la provincia del Tungurahua con el 17\% (161) de las cuales 133 se constituyeron jurídicamente cantón Ambato, con lo cual acapara el 82,60\%, de participación financiera en relación a toda la provincia de Tungurahua, cuya distribución per cápita es de una organización por cada 2745 habitantes, lo que demuestra la alta competitividad existente, promoviendo desarrollo y generando oportunidades en el mercado interno y externo en los tres sectores de la economía.

Los autores coinciden con el criterio de la Asociación de Organismos del Sector Financiero Popular y Solidario (ASOFIPSE), considera que no hay un número ideal al que se deba llegar en el proceso de depuración. Es indiferente el número de entidades que existan, siempre que 
tengan solvencia, prudencia financiera y compromiso social; considerando además que entre una de las principales causas para la extinción de COAC, es que incumplieron con el objeto social; es decir, entidades que dejaron de operar de manera unilateral, o que durante un período de tiempo determinado y de manera injustificada no presentaron estados financieros que dieran cuenta de su accionar y su situación real.

La mitad de las liquidaciones se ha dado por esta razón. Otra causa de liquidaciones de cooperativas es que registraron pérdidas de más del 50\% de su capital social, es decir se trata de casos en que ese monto de pérdidas no ha podido ser compensada por las reservas patrimoniales o de los mismos socios, como dueños de su cooperativa; los cual se ha visto acompañado de la existencia de inadecuados estatutos los cuales más que una solución se han constituido un problema lo cual ha generado mayor inestabilidad financiera.

Problemática que ha dado lugar para que entre el 2015 y abril del 2019 se hayan dado 106 procesos de fusión, la mayoría, por absorción de entes más grandes que se fusionan con entidades de los segmentos más pequeños; sin embargo, se destaca que a pesar de que el sector está reduciéndose en número de entidades, hay confianza por parte de la gente, porque el número de socios ha ido en aumento.

Los sectores cooperativos a marzo de este año muestran que los depósitos crecieron un 12,5\% en relación a igual mes del año pasado, según un reporte realizado por la Asociación de Bancos Privados (ASOBANCA). Con más recursos depositados, las entidades también colocaron más créditos. La cartera bruta a marzo creció un $25,4 \%$ en relación al mismo mes del 2018, dice el mismo reporte. La economía popular y solidaria también forma parte del programa que llevará a cabo el Gobierno como parte del acuerdo económico con el Fondo Monetario Internacional (FMI).

En este mismo ámbito, esta investigación enfoca su análisis en las COAC de la provincia de Tungurahua, donde del 100\% de COAC del cantón Ambato el 83\% pertenecen al segundo segmento, las mismas que presenta un promedio del $8.43 \%$ de cartera vencida $(16.055,26$ dólares aproximadamente) del total de cartera, igualmente presentan una cartera por vencer del 82,50\% (1.104.271,28 dólares aproximadamente), emitida a los clientes a través de créditos Comerciales, de Consumo, de Vivienda para la Microempresa, créditos Educativos, y de Inversión Pública; cuyo efecto si no es controlado promueve el crecimiento del índice de morosidad, según información divulgada por las SEPS en el boletín financiero del segmento 2 del primer semestre del año 2019.

Cabe destacar que, de acuerdo con los datos de la Superintendencia, desde el 2012 se han liquidado unas 129 entidades y 205 han entrado en proceso de liquidación. Entre el 2017 y 2018, en Tungurahua se registró la mayor cantidad de entidades que se liquidaron en el cantón Ambato; tales como Visión Indígena, Credipichincha Ltda, Yachak Runa Ltda, 
Stephen Hawking Ltda, Yuyak Runa Ltda.; tendencia que no es indiferente a la realidad nacional etc.

Igualmente los autores coinciden con Bedón (2015), al sustentar que los problemas de rentabilidad, liquidez y solvencia de las COAC es producto de la inadecuada administración de la cartera de créditos promueve un alto porcentaje de deudores que no han cumplido con sus compromisos, generando el incremento de la Cartera Vencida que conlleva a la reducción de la rentabilidad económica-financiera de las COAC a pesar de existir refinanciación y restructuración de cartera; de la misma forma la infidelidad de los clientes, quienes aspiran tener mayores beneficios en una recién creada y la limitada administración financiera de las entidades recién creadas ha dando lugar a la existencia de un mayor nivel de riesgo y la generación de cartera vencida.

\section{Metodología}

El desarrollo de la presente investigación se fundamenta, en métodos de nivel teórico, empírico y estadístico, como: análisis y síntesis, cualitativo - cuantitativo, histórico-lógico, enfoque de sistema, la modelación, análisis comparativo, observación científica, revisión documental, encuestas entrevistas individuales y colectivas, estadística descriptiva y el uso de análisis financieros validados tales como la metodología P.E.R.L.A.S.

\section{Resultados}

A partir de la muestra, 64 COAC del segmento dos de la provincia de Tungurahua se realizó una evaluación de la gestión financiera, considerando para ello los estados financieros del ejercicio económico 2018 correspondiente al primero y segundo semestre.

En este sentido, los resultados consolidados fueron determinados a partir del Análisis vertical y horizontal del Balance General de las COAC del segmento dos de la provincia de Tungurahua correspondiente al primer y segundo semestre del año 2018, tal como se muestra en la Tabla $\mathrm{N}^{\circ} 1$.

- Análisis Vertical: se demuestra que la cartera de crédito del primer semestre ocupa el $75 \%$ de los activos y el 78\% durante el segundo semestre constituyéndose en la mayor cantidad de los activos en ambos periodos, seguida por la cuenta de fondos disponibles con el $13 \%$ y $9 \%$ respetivamente.

- Análisis Horizontal: se puede evidenciar que los activos en forma general han decrecido en un $27 \%$, constituyéndose la cuenta de fondos disponibles como la de mayor decrecimiento con el $84 \%$ y la de mayor crecimiento es la de inversiones con el $8 \%$ 
Tabla $\mathbf{N}^{\circ} \mathbf{1}$ Analisis vertical y horizontal Balance General

\begin{tabular}{|c|c|c|c|c|c|c|}
\hline CUENTA & $\begin{array}{c}\text { I } \\
\text { semestre } \\
2018\end{array}$ & $\begin{array}{c}\text { Análisis } \\
\text { vertical } \\
\text { I Smt. } \\
2018\end{array}$ & $\begin{array}{c}\text { II } \\
\text { semestre } \\
2018\end{array}$ & $\begin{array}{c}\text { Análisis } \\
\text { rertical } \\
\text { II Smt. } \\
2018\end{array}$ & $\begin{array}{c}\text { Valores } \\
\text { ABS }\end{array}$ & $\begin{array}{l}\text { Valores } \\
\text { Relativos }\end{array}$ \\
\hline ACTIVD & $342.147,43$ & & $268.945,65$ & & $-73201,79$ & $-27 \%$ \\
\hline FONDOS DISPONIBLES & $43.624,22$ & $13 \%$ & $23.760,38$ & $9 \%$ & - $19.863,84$ & $-84 \%$ \\
\hline INVERSIONES & $15.266,61$ & 496 & 16.571 .45 & $6 \%$ & 1304,84 & $8 \%$ \\
\hline CART ERA DE CREDITOS & $257.842,31$ & $75 \%$ & $208.499,90$ & $759 \%$ & - 49342,41 & $-299 \%$ \\
\hline CUENTAS POR COBRAR & 5.224 .80 & $2 \%$ & 3337,18 & $1 \%$ & - $\quad 1887,62$ & $-57 \%$ \\
\hline BEENES REAUZABLES, Y NOUTIUZADOS POR LAINSTITUCION & 2391,90 & $1 \%$ & $2,452,36$ & $1 \%$ & 60,45 & $2 \%$ \\
\hline PROPIEDADES Y EQUIPO & $13.204,08$ & 496 & $10.958,75$ & $4 \%$ & - $\quad 2245,33$ & $-20 \%$ \\
\hline OTROS ACTIVOS & $4.593,50$ & $1 \%$ & 3365,63 & $1 \%$ & - $\quad 1227,87$ & $-369 \%$ \\
\hline TOTALACTIVO & $342.147,43$ & $100 \%$ & $268.945,65$ & $100 \%$ & $-73.201,79$ & $-27 \%$ \\
\hline GASTOS & 4. 365,33 & & $21.151,90$ & & $16.786,57$ & $79 \%$ \\
\hline TOTALACTIVO Y GASTOS & $346.512,76$ & & $290.097,54$ & & $-56.415,21$ & $-19 \%$ \\
\hline PASINO & $304.715,10$ & & $234.957,19$ & & - $69.757,92$ & $-30 \%$ \\
\hline OBLIGACIONES CONEL PUBUCD & $280.014,76$ & $92 \%$ & $211.595,65$ & $90 \%$ & - $68.419,11$ & $-32 \%$ \\
\hline OBLIGACIONES INMEDIATAS & 30,97 & O\% & 42,94 & $0 \%$ & 11,97 & $28 \%$ \\
\hline CUENTAS POR PAGAR & $5.545,87$ & $2 \%$ & $4.893,67$ & $2 \%$ & 652,21 & $-13 \%$ \\
\hline OBZIGACIONES FINANDERAS & $18.502,37$ & $6 \%$ & $17.791,25$ & $8 \%$ & 711,11 & -496 \\
\hline OTROS PASIVOS & 621,14 & $0 \%$ & 633,68 & $0 \%$ & 12,55 & $2 \%$ \\
\hline TOTAL PASIVO & $304,715,10$ & $100 \%$ & $234.957,19$ & $100 \%$ & - $69.757,92$ & $-30 \%$ \\
\hline PATRIMONIO & $37.256,68$ & & $34.484,15$ & & - 2772,53 & $-8 \%$ \\
\hline CAPITAL SOCIAL & $21.265,47$ & $57 \%$ & $16.177,56$ & $47 \%$ & - $\quad 5.087,92$ & $-31 \%$ \\
\hline RESERN AS & 13.272 .86 & $36 \%$ & $16.619,24$ & $45 \%$ & 3346,38 & $20 \%$ \\
\hline OTROS APORTES PATRIMONIALES & 2635,87 & $7 \%$ & 532,96 & $2 \%$ & - 2102,91 & $-395 \%$ \\
\hline SUPERAVIT POR VAUACIONES & 1623,19 & $49 \%$ & 1623,19 & $5 \%$ & - & $0 \%$ \\
\hline RESULTADOS & - $\quad 1.540,71$ & $-4 \%$ & 468,79 & $-1 \%$ & 1071,92 & $-229 \%$ \\
\hline TOTAL PATRIMONIO & $37.256,68$ & $100 \%$ & $34.484,15$ & $100 \%$ & - 2772,53 & $-8 \%$ \\
\hline TOTAL PASIVO Y PATRIMONID & $341,971,78$ & & $269.441,34$ & & $-72530,45$ & $-27 \%$ \\
\hline INGRESOS & 4. 540,98 & & $20.656,21$ & & $16.115,23$ & $78 \%$ \\
\hline TOTAL PASIVD, PATRIMONIDE INGRESOS & $346.512,76$ & & $290.097,54$ & & $-56.415,21$ & $-19 \%$ \\
\hline CUADRE & 0,00 & & 0,00 & & 0,00 & $470 \%$ \\
\hline CUENTAS DE ORDEN & $687.395,08$ & & $508.289,09$ & & $-179.105,99$ & $-35 \%$ \\
\hline CUENTAS DE ORDEN DEUDORAS & $82,230,49$ & $12 \%$ & $92,476,93$ & $18 \%$ & $10.246,44$ & $11 \%$ \\
\hline CUENTAS DE ORDEN ACREEDORAS & $605.164,59$ & $88 \%$ & $415.812,17$ & $82 \%$ & $-189,352,43$ & $-46 \%$ \\
\hline
\end{tabular}

Elaborado por: Los autores

- Análisis Vertical: se evidencia que, la cuenta obligaciones con el público durante el primer semestre ocupa el $92 \%$ de los pasivos y el $90 \%$ durante el segundo semestre, seguida por la cuenta obligaciones financieras con el $6 \%$ y $8 \%$ respetivamente.

- Análisis Horizontal: se puede evidenciar que los pasivos en forma general han decrecido en un $30 \%$, constituyéndose la cuenta obligaciones con el público como la de mayor decrecimiento con el $32 \%$ y la de mayor crecimiento es la cuenta obligaciones inmediatas con el $28 \%$ 
- Análisis Vertical: en el patrimonio se evidencia que, la cuenta Capital social durante el primer semestre ocupa el $57 \%$ del patrimonio y el $47 \%$ durante el segundo semestre, seguida por la cuenta Reservas con el $36 \%$ y $48 \%$ respetivamente.

- Análisis Horizontal: se puede evidenciar que el Patrimonio en forma general han decrecido en un 8\%, y la cuenta Otros Aportes Patrimoniales como la de mayor decrecimiento con el $395 \%$.

En este mismo ámbito, se evalúa el Estado de Resultados de las COAC del segmento dos de la provincia de Tungurahua correspondiente al primer y segundo semestre del año 2018, tal como se muestra en la Tabla $\mathrm{N}^{\circ} 2$.

Tabla $\mathbf{N}^{\circ} 2$ Analisis vertical y horizontal Estado de Resultados

\begin{tabular}{|c|c|c|c|c|c|c|}
\hline CUENTA & $\begin{array}{c}\text { I semestre } \\
2018\end{array}$ & $\begin{array}{l}\text { Análisis } \\
\text { vertical } \\
\text { I Smat. } \\
2018\end{array}$ & $\begin{array}{c}\text { II semestre } \\
2018\end{array}$ & $\begin{array}{l}\text { Análisis } \\
\text { vertical } \\
\text { II Snt. } \\
2018\end{array}$ & $\begin{array}{c}\text { Valores } \\
\text { ABS }\end{array}$ & $\begin{array}{c}\text { Valores } \\
\text { Relativos }\end{array}$ \\
\hline ITIAL INGRESOS & $4,540,98$ & & $20,656,21$ & & 1611523 & $78 \%$ \\
\hline NTERESE Y DESCUENIOS GANADOS & $4.038,22$ & 896 & $10,504,10$ & 996 & $15,455.88$ & $79 \%$ \\
\hline NTERESE CAUSADOS & $1.751,02$ & $39 \%$ & $8.897,52$ & $43 \pi$ & $7.145,49$ & $80 \%$ \\
\hline NARG EN NETOINTERESE & $2.280,20$ & $50 \%$ & $10,606,59$ & 518 & 8312,39 & $78 \%$ \\
\hline NGRESOS PORSERVIOOS & 119,02 & $\mathbb{Z}_{6}$ & $4 \mathbb{Z}, 17$ & $2 \%$ & 283.15 & $70 \%$ \\
\hline CON SIONES CAUSADAS & 0,00 & $\sigma_{6}$ & 18,28 & $0 \%$ & 1828 & $100 \%$ \\
\hline UTUDADES FINANOERAS & 0,08 & $\sigma_{6}$ & 34.50 & $0 \%$ & 1441 & $99 \%$ \\
\hline NARG EN BRUTIDFINANOERO & $2.500,28$ & 53 & $11.194,72$ & 545 & 8687,44 & $78 \%$ \\
\hline PROVISIONES & 57273 & $13 \times 6$ & $2.639,17$ & $13 \%$ & 2066,44 & $78 \%$ \\
\hline MARG EN NETOFINANA ERO & $1.934,55$ & $4 \mathrm{Z}_{6}$ & $8.555,55$ & $4 \mathrm{BZ}$ & 662100 & $77 \%$ \\
\hline GASTOS DE OPERACON & 1.854 .92 & 4186 & $9.173,20$ & 445 & 730828 & $80 \%$ \\
\hline NARGEN DEINTERRMELACON & 69,63 & $Z_{6}$ & 617,65 & $-3 \%$ & 687,29 & $111 \%$ \\
\hline OTROS ING RESOS OPERACONALES & 9,90 & $\sigma_{6}$ & $\mathbf{Z}, 82$ & $0 \%$ & 1892 & $66 \%$ \\
\hline NARG EN OPERAOONAL. & 7953 & $\bar{Z}$ & 586,83 & $-3 \%$ & 66837 & $114 \%$ \\
\hline ORTOS NG RESOS & 27278 & $\mathrm{~B}_{6}$ & 515,88 & $3 \%$ & 24410 & $47 \%$ \\
\hline ORT05 GASTOSY PERDDAS & 15878 & 36 & 68,40 & $0 \%$ & 9038 & $-132 \%$ \\
\hline GANANCA O (PERDDA) ANTE DE IVPUESTOS & 19353 & 46 & 140,36 & $-1 \%$ & 333,89 & $28 \%$ \\
\hline INPUESTOSY PARTICPACONA EVPLEADOS & 17,83 & $\sigma_{6}$ & 355,33 & $2 \%$ & 337,45 & $95 \%$ \\
\hline GANANCAO (PERD DA) DELEIERCIOO & 175,65 & 46 & 495,69 & $-2 \%$ & 67134 & $135 \%$ \\
\hline
\end{tabular}

Elaborado por: Los autores

- Análisis Vertical: a partir de los ingresos totales, en este sentido se puede decir que en el primer semestre el Margen Bruto Financiero es del 55\% y en el siguiente periodo del 54\%, destacándose que los Gastos Operacionales en el primer semestre son el $44 \%$ y en el segundo $44 \%$, destacándose que durante el primer semestre existe ganancia y en el segundo perdida al final del ejercicio.

- Análisis Horizontal: el ingreso del primer semestre en relación al segundo semestre se incrementó en $78 \%$, pero los gastos también se incrementaron en un $80 \%$ con lo 
cual se justifica que en ese periodo exista pérdidas durante el segundo semestre del año 2018.

Los datos obtenidos del análisis horizontal y vertical del Estado de Pérdidas y Ganancias, y del Balance General del periodo 2018 se complementan con el análisis PERLAS el cual considera información financiera 2017 tal como se muestra en la Tabla $\mathrm{N}^{\circ} 3$

Tabla $\mathbf{N}^{\circ} \mathbf{3}$ Analisis PERLAS

\begin{tabular}{|c|c|c|c|c|c|c|}
\hline Area & PERLAC & Descripiór & Metw & คiñ 2017 & Ä̃o 201B & Cafficarión \\
\hline \multirow{3}{*}{$P=P B O T E C C I O N$} & $\mathrm{P} 1$ & $\begin{array}{c}\text { Provisión para préstamos inoobrables / } \\
\text { Provision reque rida para préstamos monosos }>12 \\
\text { meses }\end{array}$ & $100 \%$ & $107 \%$ & $78 \%$ & Debil \\
\hline & $\mathrm{P} 2$ & $\begin{array}{l}\text { Provision neta para prestamos } \\
\text { incobrables/Provisión reque rida para } \\
\text { prestamos morosos de } 1 \text { a } 12 \text { meses }\end{array}$ & $35 \%$ & $6 \%$ & $7 \%$ & Debil \\
\hline & PE & Solvenia & $y=111 \%$ & $87 \%$ & Debil & Debil \\
\hline \multirow{6}{*}{$\begin{array}{c}\text { E= } \\
\text { ETRUCTURA } \\
\text { FINANCIERA } \\
\text { EFICAZ }\end{array}$} & E1 & Piestamos Netos/Actiwo Total & $70-80 \%$ & $83 \%$ & $87 \%$ & Debil \\
\hline & E2 & Imersiones Liquidas/Act wo Total & $c=16 \%$ & $4 \%$ & $6 \%$ & Fuerte \\
\hline & E & Imersiones Financieras/Activo Tota & $c=28$ & $4 x$ & $6 \%$ & Debil \\
\hline & E5 & Depositos de Ahorro/Activo Total & $70-8008$ & $1 \exists \%$ & $9 \%$ & Debil \\
\hline & $E 7$ & Aportaciones/Actiwo Total & $c=80 \%$ & $6 \%$ & $6 \%$ & Fuerte \\
\hline & E8 & Capital Institucional/Activo Total & $r=10 \%$ & 6\% & $6 \%$ & Debil \\
\hline \multirow{3}{*}{$\begin{array}{l}\text { R }=\text { TASAS DE } \\
\text { RENDINIENTO Y } \\
\text { COSTOS }\end{array}$} & R\& & $\begin{array}{c}\text { Margen Bruto/Promedio Actiwo } \\
\text { Total }\end{array}$ & $\begin{array}{c}\text { Variable } \\
\text { relacionada } \\
\text { con } \\
\text { el } \\
\text { cumplimiento } \\
\text { defg } \\
\end{array}$ & 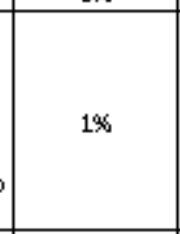 & $2 \%$ & Debil \\
\hline & $\mathrm{R} 9$ & $\begin{array}{c}\text { Gastos Operativos/Promedio Actiwo } \\
\text { Total }\end{array}$ & $c=5 \%$ & $0,39 \%$ & $1,92 \%$ & Fuerte \\
\hline & R10 & $\begin{array}{c}\text { Provisiones Activos de } \\
\text { Riesgo/Promedio Actiwo Total }\end{array}$ & $\begin{array}{l}\text { "P1 }=100 \% \\
\text { "P } 2=35 \%\end{array}$ & $0,06 \%$ & $0,10 \%$ & Debil \\
\hline L= LIQUIDEZ & L] & $\begin{array}{l}\text { Activos Liquidos } \\
\text { |mproductivos/Activo Total }\end{array}$ & $<1 \%$ & 18. & 13. & Debil \\
\hline \multirow{2}{*}{$\begin{array}{l}A=C A L I D A D D E \\
\text { LOS ACTIYOS }\end{array}$} & A1 & MorosidadTotal/Cartera Biuta & $c=5 \%$ & $4 x$ & $7 \%$ & Debil \\
\hline & A2 & Activos /mproductiwos/Actiwo Total & $c=5 \%$ & 118 & $12 \%$ & Debil \\
\hline \multirow{6}{*}{$\begin{array}{l}\text { S=SEN̂ALES DE } \\
\text { CRECIMIENTO }\end{array}$} & 51 & Crecim ientode prestamos & "E1=70-80\% & $22 \%$ & $23 \%$ & Debil \\
\hline & 52 & Crecimientode inversiones liquidas & ${ }^{\circ} \mathrm{E} 2 c=16 \%$ & $8 x$ & $9 \%$ & Fuerte \\
\hline & 55 & Crecimientode depositos de ahorro & "E5 $=70-80 \%$ & $\pi \%$ & $\pi \%$ & Debil \\
\hline & 57 & Crecimientode aportaciones & ${ }^{n} E 7 r=20 \%$ & $-31 \%$ & $-31 \%$ & Fuerte \\
\hline & 58 & Crecimie nto de capital institucional & "E\& $s=10 \%$ & $-31 \%$ & $-31 \%$ & Fuerte \\
\hline & 511 & Crecimientodel activo total & $\begin{array}{c}\text { SInflacion+10 } \\
\%\end{array}$ & $8 \times$ & $8 \times$ & Debil \\
\hline
\end{tabular}

Elaborado por: Los autores

a) Puntos débiles detectados a partir del análisis Perlas

- En las Cooperativa de Ahorro y Crédito del segundo segmento de la provincia de Tungurahua no existe una adecuada política para provisiones para préstamos 
incobrables, todos los indicadores relacionados a la protección son débiles por ejemplo: en corto plazo 78\% y largo plazo 7\%; es decir existe un amplio margen en relación las metas del 100\% y 35\%; igualmente la rentabilidad es del $87 \%$ inferior a la meta establecida del $111 \%$.

- La relación que existe entre la cartera de préstamos netos y el total de activos $87 \%$ es superior lo estimado en las metas $70 \%-80 \%$

- Las inversiones financieras entorno al total activos es débil presenta deficiencias es del $6 \%$ superior a la meta establecida del $<=2 \%$

- La relación que existe entre depósitos de ahorro y el total de activos $9 \%$ es superior lo estimado en las metas $70 \%-80 \%$

- La relación que existe entre Capital Institucional y el Activo Total 6\% es superior lo estimado en las metas $>=10 \%$

- Cuando se establece una comparación del Margen Bruto en relación al promedio del activo, es del $2 \%$ lo cual es débil porque no se cumple con la estructura de eficiencia del Capital Institucional Neto

- La tasa de rendimiento y Costos; respecto de la Provisión de Activos, sobre el promedio del Activo Total se muestra débil ya que puede responder a las provisiones de préstamos incobrable en el corto y largo plazo

- La liquidez es débil porque los Activos líquidos improductivos en relación al activo total $1 \%$ cuando la meta tenía que ser menor al $1 \%$, lo cual quiere decir que existe fondos líquidos inactivos.

- Las cooperativas del Segmento dos, tienen activos improductivos y un alto índice de morosidad, lo cual se evidencia al comparar las metas con los resultados encontrados así, por ejemplo; la relación entre la Morosidad Total y la Cartera Bruta es del 7\%, y la meta establecida, cuya meta es $<=5 \%$, de igual forma la relación Activos Improductivos con el Activo Total es del 12\% cuando la meta establecida es del $<=5 \%$

- Entorno al crecimiento de préstamos el índice es del $23 \%$ lo cual es bueno, pero al contrarrestar estructura financiera eficaz existe problemas porque los prestamos netos en relación a los activos son del $87 \%$ 
- El crecimiento de depósito en ahorro sigue siendo débil a pesar que en porcentaje representa el 77\%, porque la relación entre los depósitos y los activos apenas representa el 9\%

- El crecimiento del Activo Total es débil porque está por debajo de la tasa vigente de mercado.

b) Puntos fuertes detectados a partir del análisis Perlas

- La relación entre las Inversiones Liquidas y el Activo Total es fuerte porque el índice es del $6 \%$ inferior a la meta establecida $<=16 \%$

- Otro punto fuerte es la relación existente entre las aportaciones y el Activo Total al ser su índice solo del $6 \%$ por debajo del parámetro meta $<=20 \%$

- El índice de la razón gastos operativos entorno al promedio total del Activo es fuerte porque está por debajo de la meta establecida, $1,92 \%$ es $<=5 \%$

- El crecimiento se muestra favorable entorno al de Inversiones Liquidas y del Activo Total es inferior al $<=16 \%$ por lo cual el crecimiento es favorable y se muestra como punto fuerte

\section{Conclusiones}

- De forma general se pude establecer que en los seis parámetros que conforma el análisis Perlas, han ayudado a determinar que existe más puntos débiles que fuertes en las Cooperativas de ahorro y Créditos del segundo segmento, lo cual promueve que se genere estrategias para cambiar esta realidad existente, y evitar los activos improductivos productos de carteras vencidas que han entrado en morosidad.

- Los puntos fuertes dan a conocer que existe tendencia de crecimiento del sector, se ve contrarrestada por la mala gestión financiera, que promueve el incremento de los activos improductivos, y la falta de provisión para préstamos en el corto plazo y largo plazo.

- La información del análisis Perlas al contrarrestar con el análisis horizontal y vertical permite determinar las causas para que durante el segundo semestre del año 2018 exista decremento en relación al semestre, y un incremento del gasto en relación al monto de ingresos.

\section{Referencias bibliográficas.}

Guzmán C.: (2006) Guía Rápida Ratios Financieros y Matemáticas de la Mercadotecnia, Edición electrónica gratuita. Texto completo en www.eumed.net/libros/2006a/ 
ARGUEDA R., y otros (Madrid 2001), "Introducción a la gestión financiera de entidades sin ánimo de lucro", Universidad Nacional de Educación a Distancia, ISBN: 84-362-4288-2

BIEMAN Harold, (1987), "Planeación Financiera Estratégica”, Editorial CECSA, México.

Bedón A. (2015) LA CARTERA DE CRÉDITOS Y LA RENTABILIDAD DE LAS COOPERATIVAS DE AHORRO Y CRÉDITO DEL SEGMENTO 2 DE LA CIUDAD DE AMBATO"

ORTEGA A.(2008). Planeación Financiera Estratégica. (1ra ed). México: Editorial McGRAW-HILL/INTERAMERICANA EDITORIES, S.A

REYES M. (2015) Finanzas para la nueva economía, El riesgo del crédito [Consulta: 8 de julio de 2018] ISBN 84-96477-76-2

YÉPEZ R. (2015) el índice de morosidad es el número de veces que representan los créditos improductivos con respecto a la cartera de créditos menos provisiones

ROSS J. (2001) Fundamentals of corporate finance (sixth edition). Irwin Mc Graw Hill, USA YEPES \& Fuente 2014, Análisis de la solidez del sistema financiero ecuatoriano, octubre 2014 [Consulta: 7 de julio de 2015] artículo de la revista Observatorio Economía Latinoamericana. Indexada en latindex con ISSN: 1696-8352, Disponible en: http://www.eumed.net/cursecon /ecolat/ec/2015/sector-financiero.html

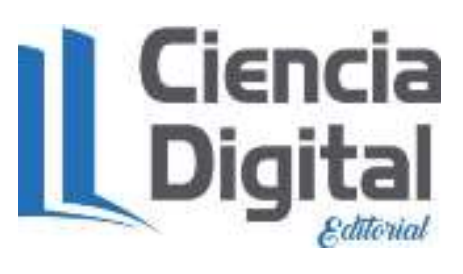




\section{PARA CITAR EL ARTÍCULO INDEXADO.}

Fonseca Vasconez, J., Tapia Bonifaz, A., Jácome Tamayo, S., \& Avalos Peñafiel, V. (2019). Evaluación económica y financiera de las Cooperativas de Ahorro y Crédito del Ecuador. Caso Segmento 2 del cantón Ambato provincia de Tungurahua. Ciencia Digital, 3(3.2), 113-121. https://doi.org/10.33262/cienciadigital.v3i3.2.718

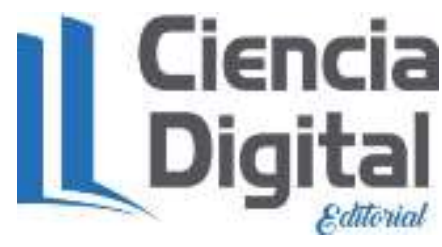

El artículo que se publica es de exclusiva responsabilidad de los autores y no necesariamente reflejan el pensamiento de la Revista Ciencia Digital.

El artículo queda en propiedad de la revista y, por tanto, su publicación parcial y/o total en otro medio tiene que ser autorizado por el director de la Revista Ciencia Digital.
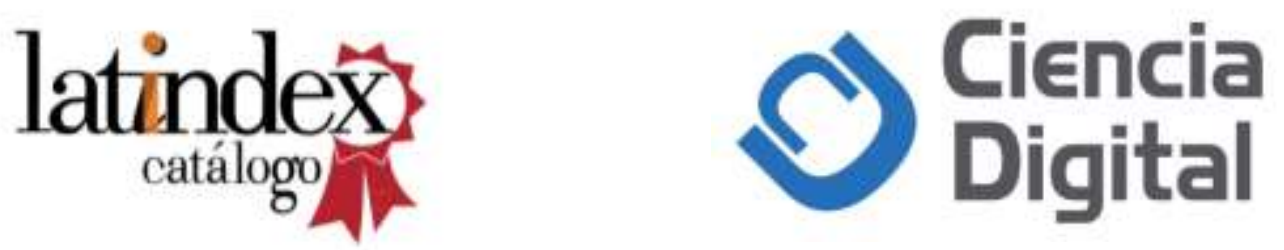